\title{
Time trends and predictors of laboratory-confirmed recurrent and severe Clostridioides difficile infections in Manitoba: a population-based study
}

\author{
Seth R. Shaffer MD MS, Zoann Nugent PhD, Andrew Walkty MD, B. Nancy Yu PhD, Lisa M. Lix PhD, \\ Laura E. Targownik MD MSHS, Charles N. Bernstein MD, Harminder Singh MD MPH
}

\section{Abstract}

Background: Many previous studies of Clostridioides difficile infection (CDI) epidemiology have used hospital discharge data codes, which can have limited accuracy. We used a data set of laboratory-confirmed cases of CDI in the province of Manitoba, Canada, to describe the epidemiology of CDI over a decade.

Methods: We conducted a population-based historical cohort study using Manitoba Health's population-wide laboratory-based CDI data set linked to administrative health databases. All individuals living in Manitoba and experiencing a CDI episode between 2005 and 2015 were included $(n=8471)$ and followed up from CDI diagnosis. We assessed time trends of CDI, incidence and predictors of recurrence and severe outcomes, and health care encounters after CDI diagnosis. CDI episodes were stratified by community versus hospital site of acquiring CDI.

Results: Between 2005 and 2009, overall CDI diagnoses decreased by an average of 12.6\% per year (95\% confidence interval [Cl] -4.4 to -20.0 ), with no statistically significant change from 2010 to 2015 . In stratified analysis, incident and recurrent CDI had a similar decrease in the initial study time period and then stabilized. The proportion of community-associated CDI cases increased by an average of $4.8 \%$ per year $(95 \% \mathrm{Cl} 2.8$ to 6.8$)$ during the study period. $\mathrm{CDI}$ acquired in a health care facility had a higher recurrence rate and more severe outcomes. Recurrence of CDI increased the likelihood of admission to hospital.

Interpretation: Between 2005 and 2015, the rates of overall laboratory-confirmed CDI, incident CDI, recurrent CDI and severe outcomes following CDI initially decreased before stabilizing, and an increasing proportion of CDI cases were community-associated. There is an increasing need to test for CDI among outpatients with diarrhea and to increase efforts to prevent recurrent CDI.

lostridioides difficile remains the most frequently reported nosocomial infection and can lead to substantial morbidity and mortality. ${ }^{1}$ In a prior study from Manitoba, Canada, Lambert and colleagues ${ }^{2}$ identified 1006 cases of C. difficile infection (CDI) over 1 year (between July 2005 and June 2006), with $51 \%$ of the cases acquired in a health care facility (HCF) and $27 \%$ acquired in the community.

Many previous studies about the epidemiology of CDI in North America have used the International Classification of Diseases (ICD) codes for CDI from administrative health or hospital discharge databases, or databases from a single institution to determine the occurrences or trends of CDI. There are caveats to using the ICD codes for CDI from hospital discharge abstract databases, as they have a low sensitivity, resulting in about $30 \%$ of laboratory-confirmed CDI cases in hospitals being missed. ${ }^{3}$ Moreover, the epidemiological patterns assessed using these codes versus laboratory-confirmed cases can be quite different. ${ }^{4,5}$ In addition, hospital discharge data do not include many community-associated cases and often cannot distinguish onset in the community versus occurrence in hospital.

Understanding population time trends of CDI incidence and recurrence is key to understanding its morbidity. We used a population-based data set of laboratory-confirmed CDI in the province of Manitoba, Canada, to study the epidemiology of CDI over a 10-year period (2005-2015), including incidence

Competing interests: See the end of the article.

This article has been peer reviewed.

Correspondence to: Harminder Singh, Harminder.singh@umanitoba.ca CMAJ Open 2020. DOI:10.9778/cmajo.20190191 
and predictors of recurrence, severe outcomes, health care encounters after CDI diagnosis and community v. hospital associated CDI.

\section{Methods}

\section{Setting and population}

Manitoba is a central Canadian province that had a population of 1.36 million people in 2018. ${ }^{6}$ Manitoba Health, Seniors and Active Living (MHSAL) is the provincial agency that oversees the delivery of universal health care in the province. Laboratoryconfirmed CDI cases among individuals 18 years or older in the entire province of Manitoba between July 2005 and December 2015 were included in this study.

\section{Data sources}

Manitoba Health, Seniors and Active Living maintains several administrative databases to monitor health care services and for reimbursement to health care providers for the services rendered. These include admissions to hospital, Discharge Abstract Database, physician claims data set and the Drug Programs Information Network database (that includes all outpatient dispensed prescriptions). Several prior studies have validated the accuracy of data in MSHAL databases, which have been used for many epidemiological studies. ${ }^{7-10}$

The MHSAL Public Health Branch Epidemiology and Surveillance Unit has maintained a population-based CDI database since 2005, developed from the legally mandated universal reporting of documented CDI cases in the province to the unit. The MHSAL CDI data set includes identifying information on individuals with CDI, including their personal health identification number (PHIN), postal code of residence at the time of CDI diagnosis, the date the stool specimen was collected and the results reported. Only loose stool, which takes the shape of its container, is tested by the Manitoba laboratories, thereby minimizing the detection of asymptomatic carriers (estimated to be $2 \%-7 \%$ of the population ${ }^{11,12}$ ).

Between July 2005 and May 2013, Manitoba laboratories performed immunoassays for the glutamate dehydrogenase antigen and $C$. difficile toxins $\mathrm{A}$ and $\mathrm{B}$, followed by the cytopathic effect assay (using viable human fibroblasts) or culture for discordant results (e.g., positive glutamate dehydrogenase antigen and negative $C$. difficile toxin A \& B immunoassay). ${ }^{13,14}$ Since May 2013, 3 laboratories (responsible for about $70 \%$ of the testing) implemented a nucleic acid amplification test (the Illumigene assay, Meridian Biosciences) for confirmation of glutamate dehydrogenase antigen-positive samples. ${ }^{14}$

Since 1984, all individuals in the province are assigned a unique PHIN. We used scrambled anonymized PHINs to link all databases included in the current study.

\section{Study measures}

We used previously recommended and used definitions for $\mathrm{CDI}^{2,15}$ (listed in Appendix 1, Supplementary Table A, available at www.cmajopen.ca/content/8/4/E737/suppl/DC1) to determine whether it was acquired in the community or in an $\mathrm{HCF}$ and whether it was incident or recurrent. Index date was defined as the date stool specimen was collected. Communityassociated CDI refers to CDIs that were likely acquired in the community. Hospital-associated CDI refers to the CDIs likely acquired in the hospitals, including those manifesting in the hospital (health care facility [HCF] onset, HCF-associated $\mathrm{CDI}$ ) or after hospital discharge (community-onset HCFassociated CDI).

The Socioeconomic Factor Index (SEFI) was used to assign socioeconomic status to each case. The SEFI is based on the neighbourhood of residence on the index date using a validated measure from Statistics Canada Census data. ${ }^{16}$ Comorbidity burden was determined from ambulatory care and hospital admission diagnoses in the year before index CDI using the Charlson Comorbidity Index (CCI) score. ${ }^{17}$ Specific common comorbid medical conditions that increase the risk of CDI (diabetes, renal disease) were used as separate covariates and were not included in the CCI for the analysis of predictors of recurrence and severe CDI. ${ }^{18-20}$ Inflammatory bowel disease (IBD) was identified using a definition based on previously validated administrative data. ${ }^{10}$

\section{Statistical analysis}

We calculated the overall incidence and recurrence of $\mathrm{HCF}$ associated; HCF-onset HCF-associated, community-onset HCF-associated, and community-associated CDI per 100000 person-years of follow-up.

We used Joinpoint software (version 4.5.0.1) ) $^{21}$ to assess CDI time trends. Unadjusted and direct age-adjusted (to the 2006 Canadian population; ${ }^{22}$ as rates have been reported to increase with age) rates were calculated. ${ }^{23}$ Time trends were expressed as annual percent change in rates with $95 \%$ confidence intervals (CIs). ${ }^{24}$ The models incorporated estimated standard errors of the age-adjusted rates. The joinpoint regression analysis approach aims to break a trend over time into 2 or more piecewise linear sections and identify the years in which the slopes change over time.

Potential patient-level predictors of recurrence of CDI were evaluated using a multivariable competing risks proportional hazards regression model. Death was considered the competing event with censoring at loss of health care coverage or end of the study period. Model covariates included sex, age, CDI infection type (HCF- v. community-associated), SEFI, diabetes, renal disease, IBD, length of stay in hospital (for the subgroup analysis among $\mathrm{HCF}$-onset $\mathrm{HCF}$-associated CDI) and frequency of ambulatory care visits and CCI score in the year before the index date. We performed separate analyses for the first and second recurrence.

A multivariable logistic regression model was used to estimate odds ratios for factors associated with outcome severity. Severe outcomes (death, admission to the intensive care unit, or colectomy) were identified after the index episode of CDI (first incident or recurrent CDI in the study period) in the 30 days after index CDI. Of note, no individual left the province in the 30 days after a CDI episode, and the last 30 days of the data set were not used in this analysis. Model covariates included presence or absence of recurrence, sex, 
age, CDI infection type, SEFI, diabetes, renal disease, IBD, length of stay in hospital (for the subgroup analysis among $\mathrm{HCF}$-onset HCF-associated CDI) and frequency of ambulatory care visits and CCI score in the year before the index date.

Predictors of hospital admission after communityassociated CDI were evaluated using multivariable Cox proportional hazards regression models with hospital admission as the event and a maximum follow-up period of 6 months with censoring loss of health care coverage, end of study period or out-of-hospital death as end points. Only first episodes of CDI that occurred during the study period were included in this analysis.

Patterns of hospital readmission after CDI detected in inpatients were also evaluated using multivariable Cox proportional hazards regression models with hospital readmission as the event and the maximum follow-up 6 months with censoring at loss of health care coverage, out-of-hospital death or end of the study period as end points. Only first infections diagnosed in inpatients who were subsequently discharged were included in this analysis, and the follow-up time started at the discharge date.

Data management and analyses were completed using SAS version 9.4 (SAS Institute).

\section{Ethics approval}

This study was approved by the Health Research Ethics Board at the University of Manitoba and the Health Information and Privacy Committee at Manitoba Health.

\section{Results}

Patient demographic and clinical characteristics are provided in Table 1.

During the study period (2005-2015), the overall rate of CDI was 92.7 (95\% CI 90.7 to 94.6 ), incident CDI 75.1 (95\% CI 73.4 to 76.9 ) and recurrent CDI 17.5 (95\% CI 16.7 to 18.4$)$ cases per 100000 person-years. The average rate of HCF-associated CDI was 55.0 cases (95\% CI 50.5 to 53.5), with HCF-onset HCF-associated CDI 39.0 (95\% CI 37.7 to 40.3) and community-onset HCF-associated 16.1 (95\% CI 15.2 to 16.9$)$ per 100000 person-years; communityassociated CDI was 28.0 cases (95\% CI 26.3 to 28.4 ) per 100000 person-years.

The age-standardized CDI rates were similar to the unadjusted rates: total CDI 88.6 (95\% CI 86.7 to 90.5); HCFassociated CDI 52.0 (95\% CI 50.5 to 53.5 ), with rates of HCF-onset HCF-associated CDI 36.5 (95\% CI 35.3 to 37.7 )

Table 1: Demographic and clinical characteristics of patients with incident or recurrent CDls

\begin{tabular}{|c|c|c|c|c|}
\hline \multirow[b]{2}{*}{ Characteristic } & \multicolumn{4}{|c|}{ No. $(\%)$ of patients* } \\
\hline & $\begin{array}{c}\text { All patients } \\
n=8471\end{array}$ & $\begin{array}{c}\text { Incident CDI } \\
n=6855\end{array}$ & $\begin{array}{c}\text { Recurrent CDI } \\
n=1616\end{array}$ & $p$ value $\dagger$ \\
\hline Sex, male & $3549(41.8)$ & 2905 (42.3) & $644(39.8)$ & 0.064 \\
\hline Age, median (IQR), yr & $71(55-82)$ & $70(53-82)$ & 77 (63-85) & $<0.001$ \\
\hline SEFI, median (IQR) & $\begin{array}{c}-0.08(-0.57 \text { to } \\
0.40)\end{array}$ & $\begin{array}{c}-0.07(-0.57 \text { to } \\
0.40)\end{array}$ & $\begin{array}{c}-0.10(-0.59 \text { to } \\
0.39)\end{array}$ & 0.30 \\
\hline $\begin{array}{l}\text { No. of ambulatory care visits in the year } \\
\text { preceding the index CDI, median (IQR) }\end{array}$ & $24(14-39)$ & $22(13-37)$ & $30(20-45)$ & $<0.001$ \\
\hline \multicolumn{5}{|l|}{ Charlson Comorbidity Index } \\
\hline 0 & $2339(27.6)$ & $2033(29.6)$ & $306(18.9)$ & $<0.001$ \\
\hline 1 & $1491(17.6)$ & 1199 (17.4) & $292(18.0)$ & \\
\hline 2 & $1510(17.8)$ & 1195 (17.4) & $315(19.4)$ & \\
\hline$\geq 3$ & $3131(36.9)$ & $2428(35.4)$ & $703(43.5)$ & \\
\hline Diabetes & $2434(28.7)$ & $1956(28.5)$ & $478(29.5)$ & 0.41 \\
\hline Renal disease & $1522(17.9)$ & $1208(17.6)$ & $314(19.4)$ & 0.091 \\
\hline Inflammatory bowel disease & $280(3.3)$ & $224(3.2)$ & $56(3.4)$ & 0.70 \\
\hline \multicolumn{5}{|l|}{ Type of index CDI (by location of acquisition) } \\
\hline HCF-onset HCF-associated & $3563(42.0)$ & $2824(41.1)$ & $739(45.7)$ & $<0.001$ \\
\hline Community-onset HCF-associated & 1469 (17.3) & $1133(16.5)$ & $336(20.7)$ & \\
\hline Community-associated & $2558(30.1)$ & $2259(32.9)$ & $299(18.5)$ & \\
\hline Indeterminate & $881(10.4)$ & $639(9.3)$ & $242(14.9)$ & \\
\hline
\end{tabular}


and community-onset HCF-associated CDI 15.5 (95\% CI 14.7 to 16.3 ); community-associated CDI 27.3 (95\% CI 26.3 to 28.4); incident CDI 72.2 (95\% CI 70.5 to 73.9 ); and recurrent CDI 16.4 (95\% CI 15.6 to 17.2 ) per 100000 person-years.

\section{Time trends}

The total CDI rate decreased from 2005 to 2009 by an average of $12.6 \%$ per year (95\% CI -4.4 to -20.0 ), and then had a nonsignificant average increase of $2.3 \%$ annually (95\% CI -2.2 to 6.9) from 2010 to 2015 (Figure 1). Incident CDI initially decreased an average of $16.1 \%$ annually from 2005 to 2008 (95\% CI -27.2 to -3.2$)$ and recurrent CDI an average of $11.5 \%$ per year from 2005 to 2010 (95\% CI -20.7 to -1.1 ), followed by nonsignificant increases in both. Severe cases of CDI initially decreased by an average of $16.6 \%$ per year from 2005 to 2012 (95\% CI -23.1 to
-9.6), but then increased nonsignificantly afterward (24.7\% [95\% CI -10 to 72.7$])$.

The rate of HCF-associated CDI decreased by an average of $15.9 \%$ per year from 2005 to 2009 (95\% CI -23.9 to -7.1 ), and then continued to show a $4.2 \%$ downward average annual trend thereafter (95\% CI -9.5 to 1.5 ) (Figure 1 ).

All other CDI categories showed time trends that were not statistically significant. The incidence of communityonset $\mathrm{HCF}$-associated CDI had an initial downward trend of $16.9 \%$ from 2005 to 2008 (95\% CI -32.5 to 2.2 ), and then an increasing trend of $1.7 \%$ per year from 2008 to 2015 (95\% CI -3.3 to 7.0). Community-associated CDI also had an increasing, though not statistically significant, trend of $1.7 \%$ per year from 2005 to 2015 (95\% CI -2.3 to 5.9).

As a result of these individual time trends, the proportion of community-associated CDI increased an average of $4.8 \%$

\section{A}

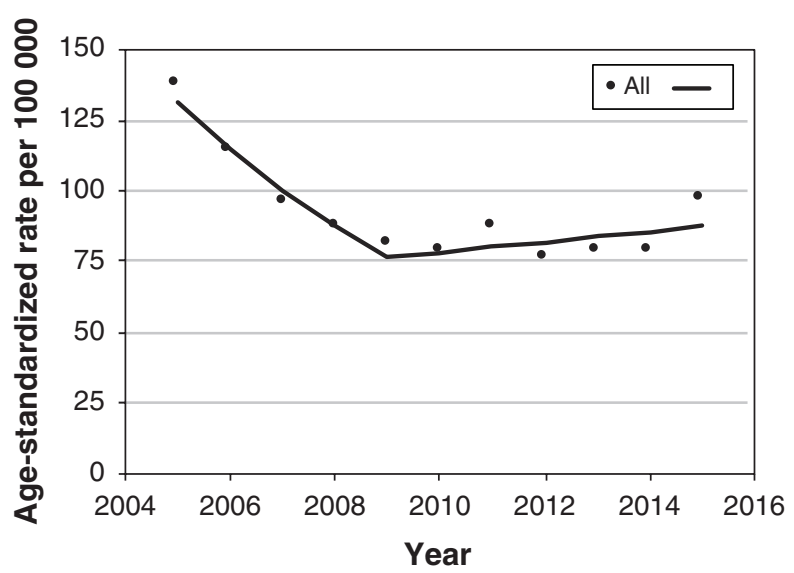

C

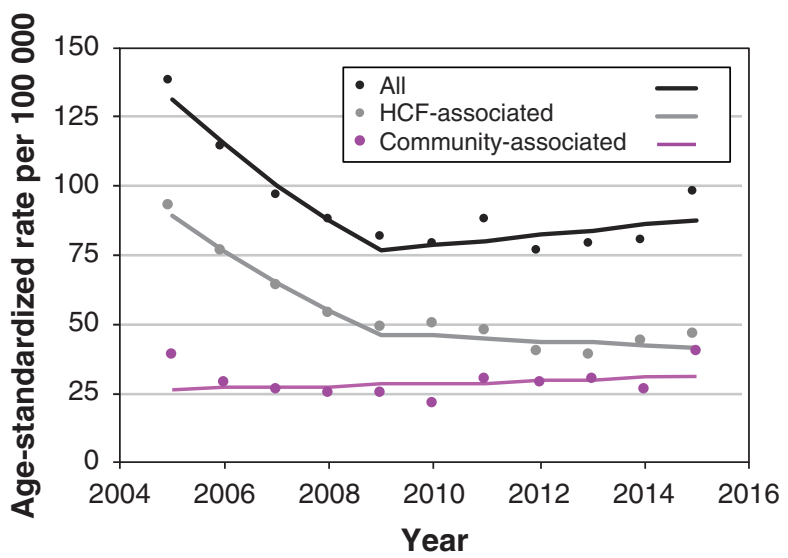

B

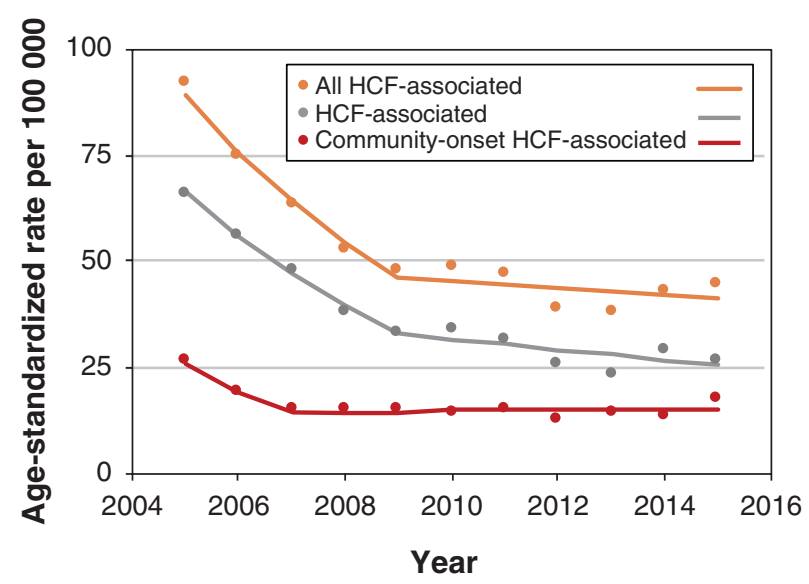

D

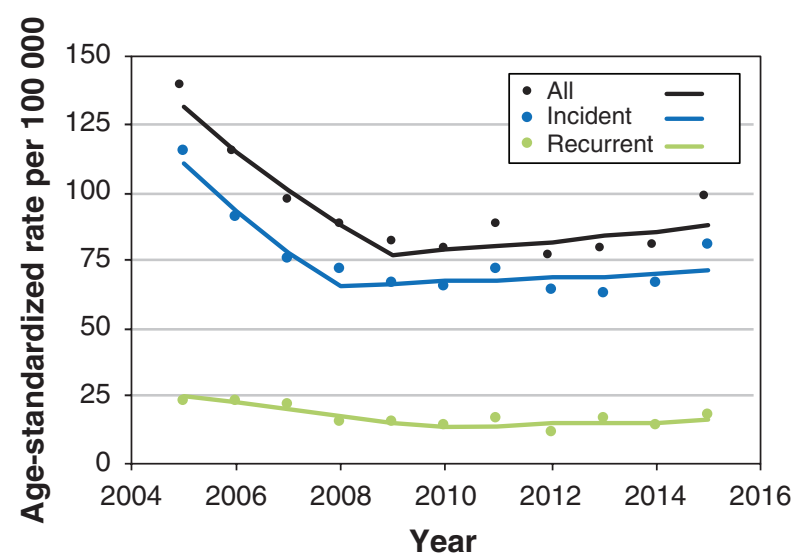

Figure 1: Age-standardized CDI rates in Manitoba, 2005 to 2015. Note: CDI = Clostridioides difficile infection, HCF = health care facility. 


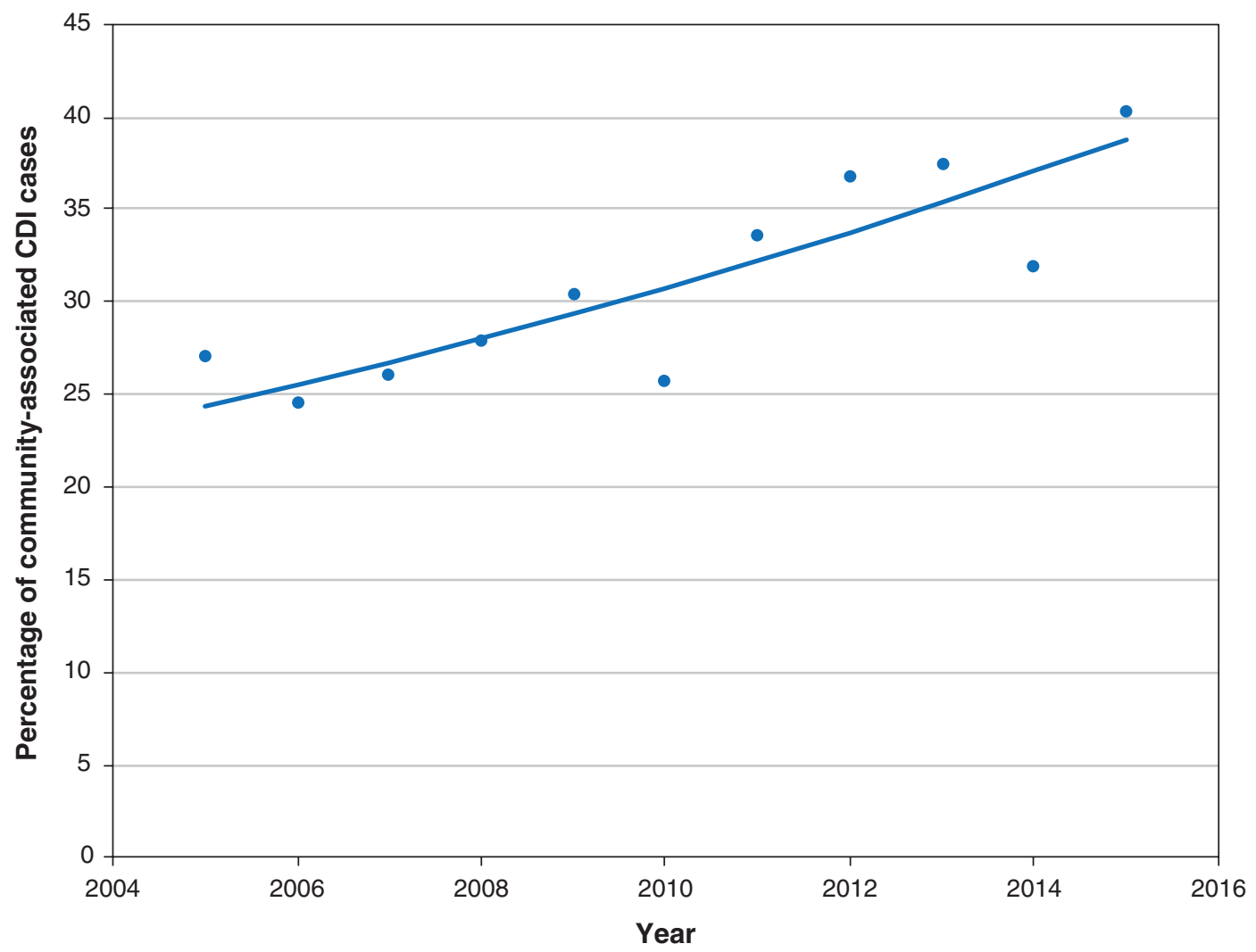

Figure 2: Proportion of CDI cases that were community-associated, 2005 to 2015. Note: CDI = Clostridioides difficile infection.

per year (95\% CI 2.8 to 6.8 ) over the duration of the study (Figure 2; Appendix 1, Supplementary Table B).

\section{Recurrent CDI}

Among 5795 patients with CDI, there were 1021 recurrences and 421 deaths within 56 days. Older age, female sex and $\mathrm{HCF}$-associated CDI were associated with increased risk of recurrent CDI (Table 2). Older age, higher frequency of ambulatory care visits in the year preceding initial CDI and community-onset $\mathrm{HCF}$-associated CDI were associated with a second CDI recurrence (Table 2).

\section{Severe CDI}

Advanced age, lower socioeconomic status, greater frequency of ambulatory care visits, increasing CCI score and diagnosis of renal disease were associated with an increased risk of a severe outcome (Table 3). Furthermore, HCF-associated CDI cases had more severe outcomes than community-associated disease.

In univariate models, there was no difference between incident and recurrent groups, but patients in the recurrent group were older, had greater comorbidity and had more frequent ambulatory care visits. When these differences were taken into account in the multivariable model, patients with recurrent cases were less likely to experience severe outcomes.

\section{Admission to hospital within $\mathbf{3 0}$ days of CDI}

About one-quarter (25.4\%) of those with incident CDI and $36.3 \%$ of those with recurrent CDI were in hospital on the index date and in the 30 days post-index date (or until death), whereas $36.7 \%$ of those with incident CDI and $35.6 \%$ of those with recurrent infection were never in hospital immediately after CDI diagnosis (no hospital admissions on or for 30 days after the index date). Of all patients, $11.2 \%$ of those with incident CDI and $10.2 \%$ of those with recurrent CDI were admitted once, whereas $3.8 \%$ of those with incident CDI and $2.5 \%$ of those with recurrent CDI were discharged and then readmitted at least once in the 30 days after the index date. The percentage of patients with incident and recurrent CDI who were in hospital over the 30 days post-index date are provided in Figure 3 and suggest a higher rate of admission to hospital for the individuals with recurrent CDI.

\section{Admission to hospital within 6 months of CDI}

The risk of hospital admission within 6 months for outpatients with CDI increased with older age, lower socioeconomic status, higher CCI score, and greater frequency of ambulatory care visits in the 1 -year period before the index date. Community-associated CDI was associated with lower rates of hospital admission than community-onset HCF- 
Table 2: Predictors of recurrent CDI among patients with diagnosis of CDI

\begin{tabular}{|c|c|c|}
\hline \multirow[b]{2}{*}{ Covariate } & \multicolumn{2}{|c|}{ Recurrence type; adjusted HR $(95 \% \mathrm{Cl})^{*}$} \\
\hline & First recurrence & Second recurrence $\ddagger$ \\
\hline \multicolumn{3}{|l|}{ Age, yr (Ref: 18-69 yr) } \\
\hline $70-79$ & $1.51(1.27$ to 1.79$)$ & 1.61 (1.14 to 2.28$)$ \\
\hline$\geq 80$ & 2.01 (1.72 to 2.34$)$ & 2.76 (2.06 to 3.70$)$ \\
\hline Female sex (v. male) & 1.16 (1.02 to 1.32$)$ & $0.99(0.78$ to 1.25$)$ \\
\hline SEFI (Ref: richer v. poorer) & $0.92(0.82$ to 1.04$)$ & 0.98 (0.78 to 1.23$)$ \\
\hline \multicolumn{3}{|l|}{$\mathrm{CCl}($ Ref: 0)§ } \\
\hline 1 & $1.23(1.02$ to 1.47$)$ & $0.83(0.57$ to 1.20$)$ \\
\hline 2 & 1.17 (0.96 to 1.42$)$ & $1.35(0.96$ to 1.91$)$ \\
\hline$\geq 3$ & $1.18(0.97$ to 1.45$)$ & $1.23(0.84$ to 1.78$)$ \\
\hline Diabetes (v. none) & $1.06(0.91$ to 1.23$)$ & $1.00(0.76$ to 1.31$)$ \\
\hline Renal disease (v. none) & $1.07(0.89$ to 1.30$)$ & $1.17(0.84$ to 1.63$)$ \\
\hline IBD (v. none) & $1.13(0.79$ to 1.63$)$ & $0.81(0.36$ to 1.83$)$ \\
\hline \multicolumn{3}{|c|}{ Ambulatory care visits in prior year (v. most) } \\
\hline Least & $1.17(0.96$ to 1.43$)$ & $1.93(1.20$ to 3.11$)$ \\
\hline Quartile 2 & $1.20(0.97$ to 1.48$)$ & $1.92(1.21$ to 3.05$)$ \\
\hline Quartile 3 & 1.10 (0.88 to 1.38$)$ & $2.12(1.37$ to 3.27$)$ \\
\hline \multicolumn{3}{|l|}{ CDI subtype (v. community-associated) } \\
\hline HCF-onset HCF-associatedף & 1.20 (1.02 to 1.42$)$ & $1.28(0.91$ to 1.79$)$ \\
\hline Community-onset HCF-associated & 1.25 (1.03 to 1.53$)$ & $1.51(1.03$ to 2.21$)$ \\
\hline Indeterminate & $1.18(0.93$ to 1.50$)$ & $1.34(0.86$ to 2.11$)$ \\
\hline \multicolumn{3}{|c|}{ 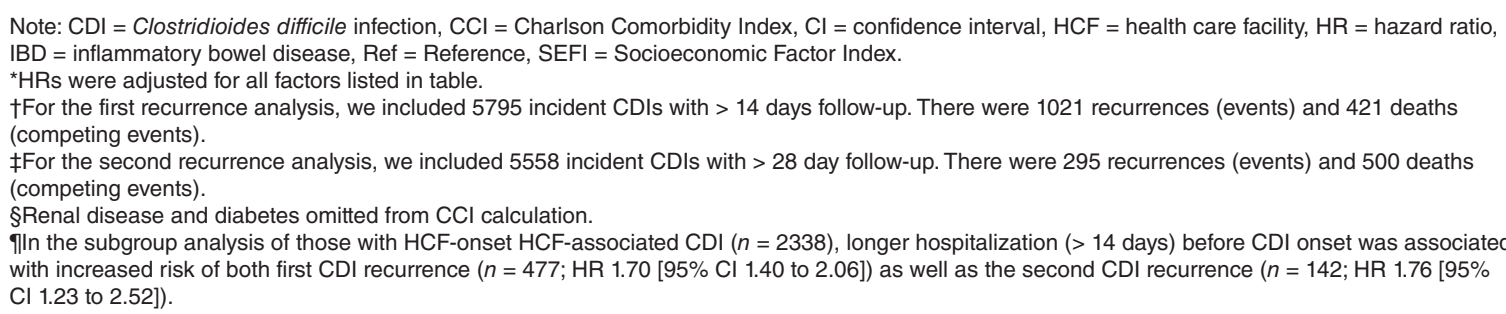 } \\
\hline
\end{tabular}

associated cases. Recurrence of CDI (v. incident infection) increased the risk of hospital admission (Table 4).

The risk of readmission within 6 months of initial discharge for all individuals admitted to hospital with CDI (incident and recurrent) who survived to discharge increased with lower socioeconomic status, greater CCI score and higher frequency of ambulatory care visits before the index date. Community-associated CDI was associated with lower rates for readmission to hospital after a hospital discharge than HCF-onset HCF-associated CDI. Recurrence of CDI increased the rate for readmission to hospital only if that recurrence happened after discharge from the original hospital admission (Appendix 1, Supplementary Table C).

\section{Interpretation}

In this population-based study using laboratory confirmed CDI cases between 2005 and 2015, the rates of overall CDI incidence, recurrent CDI and severe outcomes following
CDI all initially decreased before stabilizing. Rates of HCF-associated CDI initially decreased as well; however, community-associated CDI cases had no substantial change through the years. These patterns led to an increasing proportion of CDI cases that are community-associated. This finding reinforces the practice of testing patients not admitted to hospital with diarrhea for CDI.

There are several potential reasons for decrease in incident CDI rates from 2005 to 2008. Increased awareness of CDIassociated risk factors and infection control procedures and judicious use of antibiotics are all possible explanations for the initial decrease. Increased rates of testing could have then counterbalanced this trend and led to the stabilization of rates from 2009 onward.

Predictors of severe outcomes were advanced age, increased number of comorbidities, prior diagnosis of renal disease and HCF-onset HCF-associated CDI. Early diagnosis and prompt treatment is especially important in these groups. 


\begin{tabular}{|c|c|}
\hline Covariate & Adjusted OR $(95 \% \mathrm{Cl}) \dagger$ \\
\hline \multicolumn{2}{|l|}{ Age, yr (Ref: 18-69 yr) } \\
\hline $70-79$ & $1.52(1.22$ to 1.89$)$ \\
\hline$\geq 80$ & 2.44 (2.02 to 2.94$)$ \\
\hline Female sex (v. male) & $0.92(0.79$ to 1.08$)$ \\
\hline SEFI & $1.11(1.02$ to 1.20$)$ \\
\hline \multicolumn{2}{|l|}{$\mathrm{CCl}$ (Ref: 0)§ } \\
\hline 1 & 1.88 (1.46 to 2.43$)$ \\
\hline 2 & 2.06 (1.59 to 2.66$)$ \\
\hline$\geq 3$ & 2.55 (1.97 to 3.30$)$ \\
\hline Diabetes (v. none) & $0.98(0.81$ to 1.18$)$ \\
\hline Renal disease (v. none) & 1.65 (1.34 to 2.02$)$ \\
\hline IBD (v. none) & $0.82(0.45$ to 1.49$)$ \\
\hline \multicolumn{2}{|c|}{ Ambulatory care visits in prior year (v. most) } \\
\hline Least & 0.69 (0.52 to 0.92$)$ \\
\hline Quartile 2 & $0.80(0.64$ to 1.01$)$ \\
\hline Quartile 3 & $0.93(0.76$ to 1.13$)$ \\
\hline \multicolumn{2}{|l|}{ CDI subtype (v. community-associated) } \\
\hline HCF-onset HCF-associatedף & 2.56 (2.02 to 3.25$)$ \\
\hline Community-onset HCF-associated & $1.32(0.99$ to 1.77$)$ \\
\hline Indeterminate & $1.02(0.71$ to 1.49$)$ \\
\hline Recurrent CDI (v. incident CDI) & $0.64(0.52$ to 0.79$)$ \\
\hline \multicolumn{2}{|c|}{ 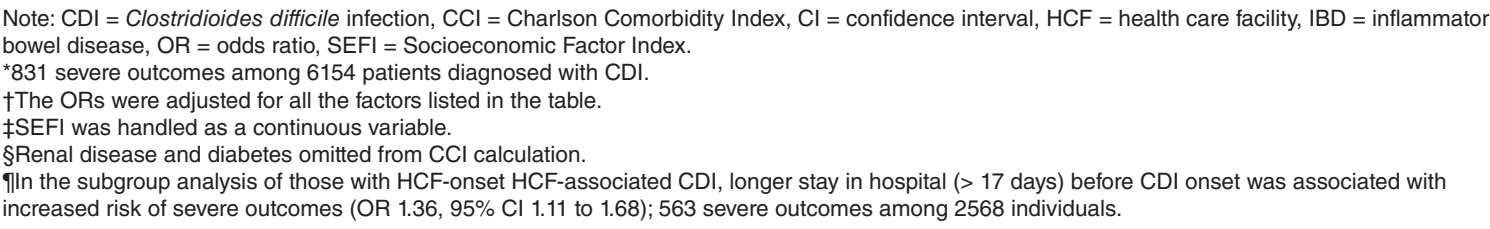 } \\
\hline
\end{tabular}

Although recurrent CDI was associated with lower risk of severe outcomes, it was associated with increased risks of admission and readmission to hospitals. This could have substantial cost implications, as costs for nosocomial CDI readmissions have been estimated to be about Can\$128 200 per hospital annually. ${ }^{25}$

There are no similar Canadian population-based studies to which we can contrast our results, although other studies have suggested an increasing occurrence of CDI over the same time period as was evaluated in this study. For example, a retrospective cohort study from the United States assessed those insured within the Optum Insight network. ${ }^{26}$ The analysis showed that the incidence of CDI increased by $42.7 \%$, from 44.1 cases for 100000 person-years in 2001 to 62.9 cases in 2012, whereas the incidence of multiple recurrent CDI also increased. A multicentre study in the US from 2000 to 2006 reported an increase in the incidence of HCF-onset HCF-associated, and community-onset HCF-associated cases of CDI, whereas the overall incidence of community-onset community-associated and indeterminate cases of CDI remained stable. ${ }^{27}$ However, this study only assessed inpatient laboratory data from 6 academic medical centres.

A Canadian study from 2009 to 2015 assessed adult inpatients from 64 acute care hospitals, and reported that HCFassociated cases of CDI decreased from 5.9 to 4.3 cases per 10000 inpatient days. ${ }^{28}$ Another population-based cohort study in Ontario, Canada assessed community-onset cases of CDI from 2003 to 2010, using ICD codes during an emergency department visit, or nonelective hospital admission with or without a colectomy. ${ }^{29}$ There were 7950 people with community-onset CDI, with a crude annual mean incidence of 7.8 per 100000 people, which is lower than that in our study. A major difference in the methodology of our study and that of previous studies is the use of a population-based laboratoryconfirmed CDI data set in our study, including in-hospital and community CDIs. The patterns discerned by the use of ICD CDI codes can be quite different than those using laboratoryconfirmed CDI cases. ${ }^{3-5}$

We identified increasing age, female sex, greater comorbidity burden and $\mathrm{HCF}$-associated $\mathrm{CDI}$ as predictors of recurrent 


\section{Research}

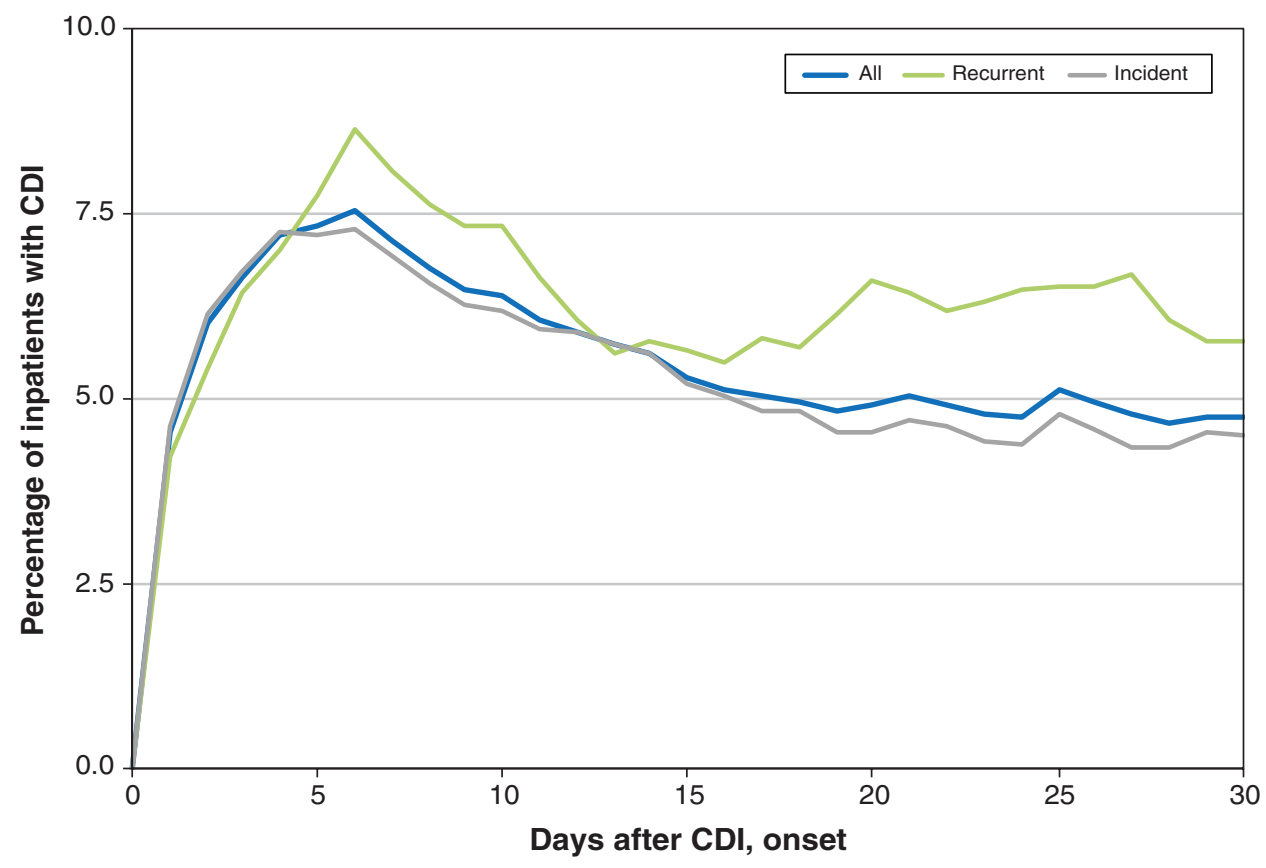

Figure 3: Inpatient status for all incident and recurrent CDI in the 30 days following laboratory confirmation of CDI among those who were outpatients at $\mathrm{CDI}$ onset. Note: $\mathrm{CDI}=$ Clostridioides difficile infection.

Table 4: Predictors of admission to hospital within 6 months for outpatients with CDI*

\begin{tabular}{|c|c|}
\hline Covariate & Adjusted HR $(95 \% \mathrm{Cl}) \dagger$ \\
\hline Recurrence (Ref: No recurrence) & $1.58(1.31$ to 1.91$)$ \\
\hline \multicolumn{2}{|l|}{ Age, yr (Ref: 18-69 yr) } \\
\hline $70-79$ & 1.55 (1.25 to 1.92$)$ \\
\hline$\geq 80$ & 1.49 (1.23 to 1.81$)$ \\
\hline Male sex (v. female) & 0.95 (0.81 to 1.11$)$ \\
\hline SEFI† & 1.17 (1.07 to 1.27$)$ \\
\hline \multicolumn{2}{|l|}{$\mathrm{CCl}($ Ref: 0)§ } \\
\hline 1 & $1.54(1.2$ to 1.96$)$ \\
\hline 2 & 1.35 (1.03 to 1.76$)$ \\
\hline$\geq 3$ & 1.83 (1.43 to 2.36$)$ \\
\hline \multicolumn{2}{|l|}{ CDI subtype (v. community-associated) } \\
\hline Community-onset HCF-associated & $1.46(1.21$ to 1.77$)$ \\
\hline Indeterminate & 1.31 (1.05 to 1.63$)$ \\
\hline \multicolumn{2}{|c|}{ Ambulatory care visits in previous year (v. least) } \\
\hline Quartile 2 & 1.34 (0.99 to 1.82$)$ \\
\hline Quartile 3 & 1.84 (1.36 to 2.48$)$ \\
\hline Most & 2.20 (1.6 to 3.02$)$ \\
\hline \multicolumn{2}{|c|}{$\begin{array}{l}\text { Note: } \mathrm{CDI}=\text { Clostridioides difficile infection, } \mathrm{CCI}=\text { Charlson Con } \\
\text { HR = hazard ratio, } \mathrm{SEFI}=\text { Socioeconomic Factor Index. } \\
{ }^{*} 307 \text { admissions among } 2530 \text { outpatients diagnosed with } \mathrm{CDI} \text {. } \\
\text { †The HRs were adjusted for all the factors listed in the table. } \\
\text { fSEFI was handled as a continuous variable. } \\
\text { §Renal disease and diabetes omitted from CCI calculation. }\end{array}$} \\
\hline
\end{tabular}


CDI, which supports previous research..$^{30}$ Renal insufficiency has also been found to be a risk factor, ${ }^{30}$ which was not shown in our study. Antibiotic use and gastric acid suppression have been reported to be associated with recurrent $\mathrm{CDI},{ }^{30,31}$ which we were unable to account for with our data as we did not have access to in-hospital medication dispensation data sets.

The lack of continued reduction in overall CDI rates is important to note in our study and suggests additional measures are necessary to develop and test to decrease the burden of this disease.

Our data support treating older individuals admitted to hospital with multiple comorbidities, who also have a higher risk of severe outcomes with CDI, with treatment modalities associated with a lower risk of recurrent CDI.

\section{Limitations}

We were unable to review individual charts to obtain other relevant clinical and laboratory information. Hence, we could not further stratify patients by the Infectious Diseases Society of America definition of CDI severity by leukocytosis or an elevated serum creatinine. ${ }^{32} \mathrm{We}$ were also unable to account for the use of antimicrobial or gastric acid suppression medications. Whether our results can be generalized to other jurisdictions will need to be determined in additional studies using population-based CDI laboratory data sets.

\section{Conclusion}

Our population-based study on CDI incidence and trends in Manitoba, Canada, suggests that although rates of CDI were initially decreasing from 2005, they have remained relatively stable for the last several years, suggesting the need for renewed focus to prevent CDI. An increased proportion of CDI cases are community associated, reflecting a decrease in HCF-onset HCF-associated CDI, perhaps because of improved hospital practices and an increasing need to test for CDI among outpatients with diarrhea. Recurrent CDI is associated with increased admission and readmission to hospital and, hence, continues to merit ongoing efforts to prevent recurrent CDI, particularly among those who do poorly with a CDI infection, such as older people admitted to hospital with multiple comorbidities.

\section{References}

1. Leffler DA, Lamont JT. Clostridium difficile infection. $N$ Engl 7 Med 2015;372:1539-48.

2. Lambert PJ, Dyck M, Thompson LH, et al. Population-based surveillance of Clostridium difficile infection in Manitoba, Canada, by using interim surveillance definitions. Infect Control Hosp Epidemiol 2009;30:945-51.

3. Singh H, Nugent Z, Yu BN, et al. Hospital discharge abstracts have limited accuracy in identifying occurrence of Clostridium difficile infections among hospitalized individuals with inflammatory bowel disease: a population-based study. PLoS One 2017;12:e0171266.

4. Singh H, Nugent Z, Yu B, et al. Assessment of Clostridium difficile epidemiology among individuals with IBD from hospital admissions databases provides a different picture than that from the laboratory confirmed diagnosis. Gastroenterology 2019;156:S-674.

5. Dubberke ER, Butler AM, Nyazee HA, et al. The impact of ICD-9-CM code rank order on the estimated prevalence of Clostridium difficile infections. Clin Infect Dis 2011;53:20-5.

6. Manitoba Health, Seniors and Active Living population report - June 1, 2019. Winnipeg: Manitoba Health, Seniors and Active Living; 2019. Available: www. gov.mb.ca/health/population/index.html (accessed 2019 July 1).
7. Kozyrskyj AL, Mustard CA. Validation of an electronic, population-based prescription database. Ann Pharmacother 1998;32:1152-7.

8. Roos LL, Mustard CA, Nicol JP, et al. Registries and administrative data: organization and accuracy. Med Care 1993;31:201-12.

9. Robinson JR, Young TK, Roos LL, et al. Estimating the burden of disease. Comparing administrative data and self-reports. Med Care 1997;35:932-47.

10. Bernstein CN, Blanchard JF, Rawsthorne P, et al. Epidemiology of Crohn's disease and ulcerative colitis in a central Canadian province: a populationbased study. Am 7 Epidemiol 1999;149:916-24.

11. Rea MC, O'Sullivan O, Shanahan F, et al. Clostridium difficile carriage in elderly subjects and associated changes in the intestinal microbiota. $\mathcal{F}$ Clin Microbiol 2012;50:867-75.

12. Galdys AL, Nelson JS, Shutt KA, et al. Prevalence and duration of asymptomatic Clostridium difficile carriage among healthy subjects in Pittsburgh, Pennsylvania. 7 Clin Microbiol 2014;52:2406-9.

13. Alfa MJ, Swan B, VanDekerkhove B, et al. The diagnosis of Clostridium difficileassociated diarrhea: comparison of Triage $C$. difficile panel, EIA for Tox A/B and cytotoxin assays. Diagn Microbiol Infect Dis 2002;43:257-63.

14. Walkty A, Lagacé-Wiens PR, Manickam K, et al. Evaluation of an algorithmic approach in comparison with the Illumigene assay for laboratory diagnosis of Clostridium difficile infection. 7 Clin Microbiol 2013;51:1152-7.

15. McDonald LC, Coignard B, Dubberke E, et al. Recommendations for surveillance of Clostridium difficile-associated disease. Infect Control Hosp Epidemiol 2007;28:140-5.

16. Chateau D, Metge C, Prior H, et al. Learning from the census: the Socioeconomic Factor Index (SEFI) and health outcomes in Manitoba. Can 7 Public Health 2012;103(Suppl 2):S23-7.

17. Charlson ME, Pompei $\mathrm{P}$, Ales KL, et al. A new method of classifying prognostic comorbidity in longitudinal studies: development and validation. 7 Chromic Dis 1987; 40:373-83.

18. Phatharacharukul $\mathrm{P}$, Thongprayoon $\mathrm{C}$, Cheungpasitporn $\mathrm{W}$, et al. The risks of incident and recurrent Clostridium difficile-associated diarrhea in chronic kidney disease and end-stage kidney disease patients: a systematic review and meta-analysis. Dig Dis Sci 2015;60:2913-22.

19. Qu HQ, Jiang ZD. Clostridium difficile infection in diabetes. Diabetes Res Clin Pract 2014;105:285-94.

20. Piper MS, Saad RJ. Diabetes mellitus and the colon. Curr Treat Options Gastroenterol 2017;15:460-74.

21. Joinpoint trends analysis software. Bethesda (MD): National Cancer Institute: Division of Cancer Control \& Population Sciences, Surveillance Research Program. Available: https://surveillance.cancer.gov/joinpoint/. (accessed 2019 Mar. 6).

22. Statistics Canada. 2006 Census of population. Available: https://www12.statcan. gc.ca/census-recensement/2006/index-eng.cfm (accessed 2020 Oct. 22).

23. Czepiel J, Dróždž M, Pituch H, et al. Clostridium difficile infection: review. Eur J Clin Microbiol Infect Dis 2019;38:1211-21.

24. Kim HJ, Fay MP, Feuer EJ, et al. Permutation tests for joinpoint regression with applications to cancer rates. Stat Med 2000;19:335-51.

25. Miller MA, Hyland M, Ofner-Agostini M, et al. Morbidity, mortality, and healthcare burden of nosocomial Clostridium difficile-associated diarrhea in Canadian hospitals. Infect Control Hosp Epidemiol 2002;23:137-40.

26. Ma GK, Brensinger CM, Wu Q, et al. Increasing incidence of multiply recurrent Clostridium difficile infection in the United States: a cohort study. Ann Intern Med 2017;167:152-8.

27. Dubberke ER, Butler AM, Yokoe DS, et al. Multicenter study of Clostridium difficile infection rates from 2000 to 2006. Infect Control Hosp Epidemiol 2010;31:1030-7.

28. Katz KC, Golding GR, Choi KB, et al. The evolving epidemiology of Clostridium difficile infection in Canadian hospitals during a postepidemic period (2009-2015). CMA7 2018;190:E758-65.

29. Nanwa N, Sander B, Krahn M, et al. A population-based matched cohort study examining the mortality and costs of patients with community-onset Clostridium difficile infection identified using emergency department visits and hospital admissions. PLoS One 2017;12:e0172410.

30. Deshpande A, Pasupuleti V, Thota P, et al. Risk factors for recurrent Clostridium difficile infection: a systematic review and meta-analysis. Infect Control Hosp Epidemiol 2015;36:452-60.

31. Song JH, Kim YS. Recurrent Clostridium difficile infection: risk factors, treatment, and prevention. Gut Liver 2019;13:16-24.

32. McDonald LC, Gerding DN, Johnson S, et al. Clinical practice guidelines for Clostridium difficile infection in adults and children: 2017 update by the Infectious Diseases Society of America (IDSA) and Society for Healthcare Epidemiology of America (SHEA). Clin Infect Dis 2018;66:987-94.

Competing interests: Harminder Singh has been on advisory boards or served as a consultant to Takeda Canada, Pendopharm, Ferring Canada, Merck Canada and Guardant Health, Inc. and has received an educational grant from Ferring Canada and research funding from Merck Canada for an independent investigator grant. Charles Bernstein has served on advisory boards or as a consultant to AbbVie Canada, Ferring Canada, Janssen Canada, Pfizer Canada, Shire Canada, Takeda Canada and Mylan Pharmaceuticals and has received unrestricted educational 
grants from AbbVie Canada, Janssen Canada, Pfizer Canada, Shire Canada and Takeda Canada. He has been on the speaker's bureau for Janssen Canada, Takeda Canada, Ferring Canada and Medtronic Canada. Laura Targownik has served on the advisory boards for Pfizer Canada, Takeda Canada, AbbVie Canada and Janssen Canada and on speaker panels for Janssen Canada, Takeda Canada and Pfizer Canada. She has received grant support from Pfizer Canada and AbbVie Canada. No other competing interests were declared.

Affiliations: Internal Medicine (Shaffer, Nugent, Walkty, Bernstein, Singh), University of Manitoba, Winnipeg, Man.; Inflammatory Bowel Disease Centre (Shaffer), University of Chicago Medicine, Chicago, Ill.; CancerCare Manitoba, Research Institute (Nugent, Singh); Community Health Sciences (Yu, Lix, Singh), University of Manitoba, Winnipeg, Man.; Division of Epidemiology and Population Health (Yu), BC Centre for Excellence in HIV/ AIDS, Vancouver, BC; Division of Gastroenterology (Targownik), Mount Sinai Hospital, University of Toronto, Toronto, Ont.

Contributors: Seth Shaffer contributed to interpretation of data and writing the original draft and editing. Zoann Nugent contributed to data curation and formal analysis and reviewing and editing of the manuscript. Charles Bernstein contributed to reviewing and editing of the manuscript. Nancy Yu, Laura Targownik and Lisa Lix contributed to critical review and editing of the manuscript. Harminder Singh contributed to conceptualization, investigation, methodology, drafting and editing of the manuscript.

Funding: Supported by a research grant from the Merck Investigator Studies Program. The opinions expressed in this paper are those of the authors and do not necessarily represent those of Merck Canada Inc. or its affiliates or related companies. The study sponsor had no role in study design, the collection, analysis and interpretation of data; the writing of the report; and the decision to submit the manuscript for publication.

Data sharing: The data used in this analysis are owned by the government of Manitoba. The authors were given permission to use the data to conduct the analysis, but do not have permission to share the data. Researchers interested in replicating the study results can apply to the ministry of health to access the data: Health Information Privacy Committee, Manitoba Health, Seniors and Active Living, 4043 - 300 Carlton Street, Winnipeg MB R3B 3M9 (email: hipc@gov.mb.ca). Instructions can be found at www.gov.mb.ca/health/hipc/submission.html.

Supplemental information: For reviewer comments and the original submission of this manuscript, please see www.cmajopen.ca/content/8/4/ E737/suppl/DC1. 